\title{
Maximum Independent Sets in Certain Powers of Odd Cycles
}

\author{
Tom Bohman * Ron Holzman ${ }^{\dagger} \quad$ Venkatesh Natarajan ${ }^{\ddagger}$
}

Submitted: Jan 2, 2008; Accepted: Jul 6, 2009; Published: Jul 24, 2009

Mathematics Subject Classification: 05C38, 05C69

\begin{abstract}
We give a complete classification of all maximum independent sets in powers of odd cycles of the form $C_{k 2^{d}+1}^{d}$.
\end{abstract}

\section{Introduction}

Consider the following natural packing problem: How many $d$-dimensional cubes of side length 2 can we pack into a $d$-dimensional torus with a fixed, odd side length? This problem can be formulated in terms of graph products as follows. If $G_{1}=\left(V_{1}, E_{1}\right)$ and $G_{2}=\left(V_{2}, E_{2}\right)$ are graphs then let $G_{1} \times G_{2}$ be the graph with vertex set $V_{1} \times V_{2}$ and an edge between distinct vertices $\left(u_{1}, u_{2}\right)$ and $\left(v_{1}, v_{2}\right)$ if and only if $u_{i}=v_{i}$ or $\left\{u_{i}, v_{i}\right\} \in E_{i}$ for $i=1,2$. The graph power $G^{d}$ is then the product of $G$ with itself $d$ times. A packing of cubes of side length 2 in the $d$-dimensional torus of side length $2 n+1$ corresponds to an independent set in $C_{2 n+1}^{d}$. (This correspondence between packings of cubes in the torus and independent sets in powers of odd cycles was first noted by Baumert et al [1]).

Let $\alpha(G)$ denote the independence number of graph $G$, i.e., the maximum size of an independent set in $G$. The independence numbers of the powers of odd cycles are also related to a central open question on the Shannon capacities of graphs. The Shannon capacity of the graph $G$ is defined as

$$
c(G)=\sup _{d}\left(\alpha\left(G^{d}\right)\right)^{1 / d}
$$

${ }^{*}$ Department of Mathematical Sciences, Carnegie Mellon University, Pittsburgh, USA. Supported in part by NSF grant DMS 0401147. E-mail: tbohman@math.cmu.edu

${ }^{\dagger}$ Department of Mathematics, Technion-Israel Institute of Technology, Haifa, Israel. Research supported by the Fund for the Promotion of Research at the Technion and by the P. and E. Nathan Research Fund. E-mail: holzman@tx.technion.ac.il

${ }_{\ddagger}^{\ddagger}$ Department of Mathematical Sciences, Carnegie Mellon University, Pittsburgh, USA. 
and gives a measure of optimal zero-error performance of an associated communication channel [6]. The odd cycles on seven or more vertices and their complements are, in a certain sense, the simplest graphs for which the Shannon capacity is not known. This follows from the Strong Perfect Graph Theorem. The Shannon capacity of $C_{5}=\overline{C_{5}}$ was determined in a celebrated paper of Lovász [5]. For a survey of zero-error information theory see [4].

The problem of determining the independence numbers of arbitrary products of odd cycles remains widely open. The best known upper bounds on these independence numbers are given (in most cases) by the Lovász-theta function $\vartheta(G)$ (which, for the sake of brevity, we do not define here) or the fractional vertex packing number $\alpha^{*}(G)$ and the simple fact

$$
\alpha(G \times H) \leq \alpha(G) \alpha^{*}(H) .
$$

The fractional vertex packing number of the graph $G$ is the maximum, over all assignments of non-negative real weights to the vertices of $G$ with the property that the sum of weights over any clique is at most 1 , of the sum of weights of the vertices of $G$. The independence numbers are known in the following cases:

$$
\begin{gathered}
\alpha\left(C_{5}^{2 j}\right)=5^{j}=\vartheta\left(C_{5}\right)^{2 j} \\
\alpha\left(C_{k 2^{d}+1}^{d}\right)=k\left(k 2^{d}+1\right)^{d-1}=k 2^{d-1}\left(\frac{k 2^{d}+1}{2}\right)^{d-1}=\alpha\left(C_{k 2^{d}+1}\right) \alpha^{*}\left(C_{k 2^{d}+1}^{d-1}\right) \\
\alpha\left(C_{k 2^{d}+3}^{d}\right)=\frac{k\left(k 2^{d}+3\right)^{d}+1}{k 2^{d}+1}=\left\lfloor\left(\frac{2 k\left(k 2^{d}+3\right)^{d-1}+1}{k 2^{d}+1}\right)\left(\frac{k 2^{d}+3}{2}\right)\right\rfloor \\
=\left\lfloor\alpha\left(C_{k 2^{d}+3}^{d-1}\right) \alpha^{*}\left(C_{k 2^{d}+3}\right)\right\rfloor
\end{gathered}
$$

Equation (1) was established in the celebrated paper of Lovász [5]. Hales [3] and Baumert et al [1] independently established (2), and Baumert et al [1] proved (3). The authors of this paper recently made progress on $\alpha\left(C_{8 k+5}^{3}\right)$ : This independence number has been determined for $8 k+5$ prime and within an additive error of 2 for arbitrary $k$ [2]. The only other power of an odd cycle for which the independence number is known is $\alpha\left(C_{7}^{3}\right)=33$ (this is established in [1] by an ad hoc argument aided by a computer search).

When the independence number is known, it is natural to ask for a description of all maximum independent sets. In addition to the inherent interest in such a characterization, it may serve as a stepping stone for obtaining upper bounds on the independence numbers of higher powers. For example, a classification of maximum and almost maximum independent sets in $C_{4 \ell+1}^{2}$ was the key to obtaining the upper bound on $\alpha\left(C_{8 k+5}^{3}\right)$ in [2]. In other related work, the authors exploited structural properties of near maximum independent sets in $C_{9}^{2}$ and $C_{9}^{3}$ to establish the upper bound $\alpha\left(C_{9}^{4}\right) \leq 350$.

In this note we give a complete classification of the maximum independent sets that achieve equality in (2). These independent sets are also the starting point for the known constructions of independent sets that achieve (3); Baumert et al established (3) by introducing an operation that transforms a maximum independent set in $C_{k 2^{d}+1}^{d}$ into a maximum independent set in $C_{k 2^{d}+3}^{d}$. 
In order to state our results we need some definitions. Throughout the paper we identity the vertex set of $C_{2 n+1}$ and $\mathbb{Z}_{2 n+1}$ in the natural way. Operations on vertices will be assumed to be over this ring unless otherwise noted. Given a set $I \subseteq[d]$ with $|I|=\ell$ and a vector $\mathbf{x} \in \mathbb{Z}_{2 n+1}^{\ell}$, the slice of $C_{2 n+1}^{d}$ given by $I=\left\{i_{1}, \ldots, i_{\ell}\right\}$ and $\mathbf{x}=\left(x_{1}, \ldots, x_{\ell}\right)$ is the set of vertices

$$
\left\{\left(v_{1}, \ldots, v_{d}\right) \in C_{2 n+1}^{d}: v_{i_{j}} \in\left\{x_{j}, x_{j}+1\right\} \text { for } j=1, \ldots, \ell\right\}
$$

Note that when we drop the coordinates in $I$ this slice projects onto the graph $C_{2 n+1}^{d-|I|}$. Furthermore, if $S$ is an independent set in $C_{2 n+1}^{d}$ then $S$ intersected with the slice maps onto an independent set in $C_{2 n+1}^{d-|I|}$ under this projection. The dimension of the slice given by $I$ and $\mathbf{x}$ is $d-|I|$.

Let $S$ be an independent set in $C_{2 n+1}^{d}$. A maximal clique $K$ in $C_{2 n+1}^{d}$ is a hole of $S$ if $K \cap S=\emptyset$. We let $H(S)$ denote the set of holes of the independent set $S$. Note that there is a natural correspondence between maximal cliques and vertices: We say that $v$ is a hole if $K_{v}:=\{v\}+\{0,1\}^{d}$ is a hole. Note that if $S_{1}$ and $S_{2}$ are independent sets in $C_{2 n+1}^{d}$ then $H\left(S_{1}\right)=H\left(S_{2}\right)$ if and only if $S_{1}=S_{2}$. (To see this, consider the set of 1-dimensional slices through a clique $K_{v}$ that is not a hole. The holes in these slices determine the location of the one vertex in $S \cap K_{v}$ by parity.) Also note that the holes in a slice of an independent set $S$ correspond to holes in $H(S)$.

We say that independent sets $S, T$ in $C_{2 n+1}^{d}$ are isomorphic, and write $S \cong T$, if there is a graph automorphism $\varphi$ of $C_{2 n+1}^{d}$ such that $\varphi(S)=T$. Note that the automorphism group of $C_{2 n+1}^{d}$ is generated by translation, negation and permutation of coordinates.

Let $k$ and $d$ be positive integers. We define a cyclic factorization of $k$ of length $d$ to be a directed cycle $\mathbf{p}=\left(p_{1}, p_{2}, \ldots, p_{d}\right)$ of positive integers such that $\prod_{i=1}^{d} p_{i}=k$. Note that, as these integers are arranged in a cycle, we have $\left(p_{1}, p_{2}, \ldots, p_{d}\right)=\left(p_{2}, p_{3}, \ldots, p_{d}, p_{1}\right)$, but we do distinguish between cycles with opposite orientations.

Now we are ready to state our classification. We begin by introducing a collection of maximum independent sets.

Lemma 1. If $\mathbf{p}=\left(p_{1}, p_{2}, \ldots, p_{d}\right)$ is a cyclic factorization of $k$ of length $d$ then there exists a maximum independent set $S_{\mathbf{p}}$ in $C_{k 2^{d}+1}^{d}$ such that

$$
H\left(S_{\mathbf{p}}\right)=\left\{\left(x_{1}, \ldots, x_{d}\right) \in \mathbb{Z}_{k 2^{d}+1}^{d}: x_{1}+\sum_{i=2}^{d}\left(\prod_{j=1}^{i-1} 2 p_{j}\right) x_{i}=0\right\}
$$

Note that the set $H\left(S_{\mathbf{p}}\right)$ defined in the Lemma, and therefore also the set $S_{\mathbf{p}}$, actually depends on the way the cyclic factorization $\mathbf{p}$ is listed. Nevertheless, using the fact that $\prod_{j=1}^{d-1} 2 p_{j+1}=-\left(2 p_{1}\right)^{-1}$ it can be checked that the set corresponding to $\left(p_{2}, p_{3}, \ldots, p_{d}, p_{1}\right)$ is isomorphic to the one corresponding to $\left(p_{1}, p_{2}, \ldots, p_{d}\right)$, and so the notation $S_{\mathbf{p}}$ is justified up to isomorphism.

Our main result is that the collection of independent sets defined in Lemma 1 is, up to isomorphism, the complete list of maximum independent sets in $C_{k 2^{d}+1}^{d}$. 
Theorem 2. If $S$ is a maximum independent set in $C_{k 2^{d}+1}^{d}$ then there exists a unique cyclic factorization $\mathbf{p}$ of $k$ of length $d$ such that $S \cong S_{\mathbf{p}}$.

The $d=2$ case of Theorem 2 was established by Baumert et al [1]. This special case plays a key role in the proof.

Before proceeding to the proofs, we establish a fact that we use throughout. Note first that if $S$ is an independent set in $C_{k 2^{d}+1}^{d}$ then the intersection of $S$ with each 1dimensional slice projects onto an independent set in $C_{k 2^{d}+1}$ and therefore contains at least one hole. It follows that any independent set in $C_{k 2^{d}+1}^{d}$ has at least $\left(k 2^{d}+1\right)^{d-1}$ holes. On the other hand a maximum independent set has exactly $\left(k 2^{d}+1\right)^{d-1}$ holes (as each vertex is in $2^{d}$ maximal cliques). It follows that a maximum independent set in $C_{k 2^{d}+1}^{d}$ has exactly one hole in each 1-dimensional slice. Consequently, the intersection of $S$ with each $\ell$-dimensional slice projects onto a maximum independent set in $C_{k 2^{d}+1}^{\ell}$.

\section{Proof of Lemma 1}

For ease of notation we set $s_{1}=1$ and $s_{i}=\prod_{j=1}^{i-1} 2 p_{j}$ for $i=2, \ldots, d$. We define an independent set $S_{\mathbf{p}}^{\prime}$ as follows:

$$
\begin{aligned}
& T_{i}=\left\{-p_{i}-1+2 j: j=1, \ldots, p_{i}\right\} \quad T=T_{1} \times T_{2} \times \cdots \times T_{d} \\
& H=\left\{\left(x_{1}, \ldots, x_{d}\right) \in \mathbb{Z}_{k 2^{d}+1}^{d}: \sum_{i=1}^{d} s_{i} x_{i}=0\right\} \quad S_{\mathbf{p}}^{\prime}=H+T .
\end{aligned}
$$

First, we show that $S_{\mathbf{p}}^{\prime}$ is an independent set. Since $T$ itself is an independent set and $H$ is a subgroup of $\mathbb{Z}_{k 2^{d}+1}^{d}$, it suffices to show

$$
H \cap\left[-2 p_{1}+1,2 p_{1}-1\right] \times \cdots \times\left[-2 p_{d}+1,2 p_{d}-1\right]=\{\mathbf{0}\} .
$$

Assume for the sake of contradiction that there is a non-zero element $\mathbf{x}=\left(x_{1}, \ldots, x_{d}\right)$ of $H$ that is also in the set $\left[-2 p_{1}+1,2 p_{1}-1\right] \times \cdots \times\left[-2 p_{d}+1,2 p_{d}-1\right]$. Let $j$ be the largest index such that $x_{j} \neq 0$. We have, working over $\mathbb{Z}$,

$$
\begin{aligned}
\left|\sum_{i=1}^{j-1} s_{i} x_{i}\right| & \leq \sum_{i=1}^{j-1} s_{i}\left(2 p_{i}-1\right) \\
& =\sum_{i=1}^{j-1} s_{i}\left(2 p_{i}\right)-s_{i} \\
& =\sum_{i=1}^{j-1} s_{i+1}-s_{i} \\
& =s_{j}-s_{1} .
\end{aligned}
$$

It follows that $0<\left|\sum_{i=1}^{j} s_{i} x_{i}\right|<k 2^{d}$, a contradiction. 
Now we consider $H\left(S_{\mathbf{p}}^{\prime}\right)$. We begin by noting that

$$
\left|S_{\mathbf{p}}^{\prime}\right|=|H| \cdot|T|=k\left(k 2^{d}+1\right)^{d-1}
$$

that is, $S_{\mathbf{p}}^{\prime}$ is a maximum independent set. It follows that $S_{\mathbf{p}}^{\prime}$ has exactly one hole in each 1-dimensional slice. Furthermore, the set of holes is symmetric with respect to $H$ : If $v \in H\left(S_{\mathbf{p}}^{\prime}\right)$ then $H+v \subseteq H\left(S_{\mathbf{p}}^{\prime}\right)$. It follows that $H\left(S_{\mathbf{p}}^{\prime}\right)$ is simply a translation of $H$, and some translation of $S_{\mathbf{p}}^{\prime}$ gives the desired independent set $S_{\mathbf{p}}$.

\section{Proof of Theorem 2}

The $d=2$ case of Theorem 2, proved in [1], plays a central role in the proof. We rephrase it as:

Lemma 3 (Baumert et al). Let $S$ be a maximum independent set in $C_{4 \ell+1}^{2}$. There exists $\alpha$ such that $\alpha \mid \ell$ and

$$
\left(t_{1}, t_{2}\right) \in H(S) \quad \Rightarrow \quad\left(t_{1}, t_{2}\right)+(2 \alpha, 1) \in H(S)
$$

Let $d \geq 3$ and let $S$ be a maximum independent in $C_{k 2^{d}+1}^{d}$. Note that, since the intersection of $S$ with any 2-dimensional slice projects onto a maximum independent set in $C_{k 2^{d}+1}^{2}$, we can apply Lemma 3 to said intersections. Thus, Lemma 3 implies that the holes in every 2-dimensional slice are a translate of some subgroup of $\mathbb{Z}_{k 2^{d}+1}^{2}$ with an appropriately chosen generator.

We now note some relations among the generators for intersecting and parallel pairs of 2-dimensional slices.

Lemma 4. Let $S$ be a maximum independent set in $C_{8 m+1}^{3}$ and let $a_{0}, \ldots, a_{8 m}, b$ be divisors of $2 m$ such that

$$
(x, y, j) \in H(S) \quad \Rightarrow \quad\left(x+2 a_{j}, y+1, j\right) \in H(S)
$$

for each $j \in \mathbb{Z}_{8 m+1}$, and

$$
(x, 0, z) \in H(S) \quad \Rightarrow \quad(x+2 b, 0, z+1) \in H(S) .
$$

Assume that $|b| \geq\left|a_{j}\right|$ for all $j \in \mathbb{Z}_{8 m+1}$. Then there exists a so that $a_{j}=$ a for all $j \in \mathbb{Z}_{8 m+1}$, and $b$ is a multiple of $2 a$.

Proof. For a positive integer $t$ and any integer $s$, let $s_{(t)}$ be the unique integer in $\{1, \ldots, t\}$ congruent to $s$ modulo $t$. Let $I_{2 t}^{+}=\{1, \ldots, t\}$ and $I_{2 t}^{-}=\{t+1, \ldots, 2 t\}$.

We consider two adjacent values of $j$, say $j=0,1$. We assume without loss of generality that $(0,0,0) \in H(S)$, that $\left|a_{0}\right| \geq\left|a_{1}\right|$, and that $b>0$. Consider the 1-dimensional slice 
$\mathbb{Z}_{8 m+1} \times\{0,1\} \times\{0,1\}$. For each integer $i \in\{1, \ldots, 4 m\}$ there is a vertex $\left(2 i, y_{i}, z_{i}\right) \in S$, where $y_{i}$ is determined by $i_{\left(2\left|a_{0}\right|\right)}$ as follows:

$$
\begin{array}{lll}
i_{\left(2\left|a_{0}\right|\right)} \in I_{2\left|a_{0}\right|}^{\operatorname{sg}\left(a_{0}\right)} & \Rightarrow & y_{i}=0, \\
i_{\left(2\left|a_{0}\right|\right)} \in I_{2\left|a_{0}\right|}^{-\operatorname{sgn}\left(a_{0}\right)} & \Rightarrow & y_{i}=1 .
\end{array}
$$

Similarly, $z_{i}$ is determined by $i_{(2 b)}$ :

$$
\begin{aligned}
& i_{(2 b)} \in I_{2 b}^{+} \Rightarrow z_{i}=0, \\
& i_{(2 b)} \in I_{2 b}^{-} \Rightarrow z_{i}=1 .
\end{aligned}
$$

We also consider the 1-dimensional slice $\mathbb{Z}_{8 m+1} \times\{0,1\} \times\{1,2\}$. Note that this slice has a hole at $(2 b, 0,1)$. For each $i \in\{b+1, \ldots, 4 m\}$ there is a vertex $\left(2 i, u_{i}, v_{i}\right) \in S$ where $u_{i}$ is determined by $(i-b)_{\left(2\left|a_{1}\right|\right)}$ :

$$
\begin{aligned}
& (i-b)_{\left(2\left|a_{1}\right|\right)} \in I_{2\left|a_{1}\right|}^{\operatorname{sgn}\left(a_{1}\right)} \Rightarrow u_{i}=0, \\
& (i-b)_{\left(2\left|a_{1}\right|\right)} \in I_{2\left|a_{1}\right|}^{-\operatorname{sgn}\left(a_{1}\right)} \Rightarrow u_{i}=1 .
\end{aligned}
$$

Let $J=\left\{4 m-\left|a_{0}\right|+1, \ldots, 4 m\right\}$. Note that for all $i \in J$ we have $i_{(2 b)} \in I_{2 b}^{-}$(since $2 b \mid 4 m$ and $\left.|b| \geq\left|a_{0}\right|\right)$ and hence $z_{i}=1$. Therefore we must have $y_{i}=u_{i}$ for all $i \in J$. As $y_{i}$ is constant for $i \in J$, it must be the case that $u_{i}$ is the same constant for $i \in J$. Since $\left|a_{0}\right| \geq\left|a_{1}\right|$, this implies that $\left|a_{0}\right|=\left|a_{1}\right|$ and one of the following two alternatives holds: either $\operatorname{sgn}\left(a_{0}\right)=\operatorname{sgn}\left(a_{1}\right)$ and $b$ is an even multiple of $\left|a_{1}\right|$, or $\operatorname{sgn}\left(a_{0}\right) \neq \operatorname{sgn}\left(a_{1}\right)$ and $b$ is an odd multiple of $\left|a_{1}\right|$. Repeating the argument for every pair of adjacent values of $j \in \mathbb{Z}_{8 m+1}$, we conclude that all $a_{j}$ have the same absolute value, and the same alternative among the two holds throughout (since $b$ is the same). But the second alternative cannot hold all around the odd cycle, so it must be the first alternative.

For a maximum independent set $S$ in $C_{k 2^{d}+1}^{d}$ and two distinct coordinates $i, j \in\{1, \ldots, d\}$, we say that the pair $(i, j)$ is aligned if there exists $\Delta_{i, j}$ such that

$$
v \in H(S) \Rightarrow v+e_{i}+\Delta_{i, j} e_{j} \in H(S),
$$

where $e_{\ell}$ denotes the $\ell$-th standard unit vector. This means that all 2-dimensional slices with coordinates $i, j$ have the same generator. Note that $\Delta_{i, j}$ is an even divisor of $k 2^{d-1}$, and that if $(i, j)$ is aligned then so is $(j, i)$ and we have $\Delta_{j, i}=\Delta_{i, j}^{-1}$.

Lemma 5. Let $S$ be a maximum independent set in $C_{k 2^{d}+1}^{d}$. Then every pair $(i, j)$ of distinct coordinates is aligned. Moreover, for any three distinct coordinates $i, j, \ell$ we have $\Delta_{i, j} \Delta_{j, \ell} \Delta_{\ell, i}=-1$.

Proof. We will prove the Lemma in the case $d=3$. The general case then follows by considering the intersection of $S$ with each 3-dimensional slice. 
Among all 2-dimensional slices in $C_{8 m+1}^{3}$, we choose one with generator $2 b$ so that $|b|$ is as large as possible. We may assume that this is the slice $\mathbb{Z}_{8 m+1} \times\{0,1\} \times \mathbb{Z}_{8 m+1}$, so that we have

$$
(x, 0, z) \in H(S) \Rightarrow(x+2 b, 0, z+1) \in H(S) .
$$

It follows from Lemma 4 that the pair $(2,1)$ is aligned.

Next, we show that alignment is contagious: once a pair is aligned, the other pairs must also be aligned. Consider for example the pair $(2,3)$. For each $i \in \mathbb{Z}_{8 m+1}$ there is a divisor $c_{i}$ of $2 m$ such that

$$
(i, y, z) \in H(S) \Rightarrow\left(i, y+1, z+2 c_{i}\right) \in H(S) .
$$

Now, fix a value of $i$. For an appropriate $j$, we have $(i, 0, j) \in H(S)$. We successively deduce that the following are holes: $(i+2 b, 0, j+1),\left(i,-2 b \Delta_{1,2}, j+1\right),\left(i, 0, j+1+4 b \Delta_{1,2} c_{i}\right)$. Since a 1-dimensional slice has only one hole, we conclude that $4 b \Delta_{1,2} c_{i}+1=0$. This uniquely determines $c_{i}$, so the value of $c_{i}$ is independent of $i$. By a similar argument, the pair $(1,3)$ is also aligned. Noting that in the above calculation we have $2 b=\Delta_{3,1}$ and $2 c_{i}=\Delta_{2,3}$, we obtain that $\Delta_{1,2} \Delta_{2,3} \Delta_{3,1}=-1$.

Since the set of holes determines the independent set, the collection $\left\{\Delta_{i, 1}: i=2, \ldots, d\right\}$ determines $S$ up to translation. By negating and permuting coordinates, we may assume $0>\Delta_{2,1} \geq \Delta_{3,1} \geq \cdots \geq \Delta_{d, 1}$. Lemma 4 then implies that $\Delta_{j+1,1}$ is a multiple of $2 \Delta_{j, 1}$ for $j=2, \ldots, d-1$. Set

$$
p_{j}= \begin{cases}\frac{-\Delta_{2,1}}{2} & \text { if } j=1 \\ \frac{\Delta_{j+1,1}}{2 \Delta_{j, 1}} & \text { if } j \in\{2, \ldots, d-1\} \\ \frac{-k 2^{d}}{2 \Delta_{d, 1}} & \text { if } j=d .\end{cases}
$$

Clearly, $\mathbf{p}=\left(p_{1}, \ldots, p_{d}\right)$ is a cyclic factorization of $k$ of length $d$. Furthermore $S_{\mathbf{p}}$ is a translation of $S$ (as the generators $\Delta_{i, j}$ are the same for both).

It remains to establish uniqueness. Let $S$ be a maximum independent set and assume $0>\Delta_{2,1} \geq \Delta_{3,1} \geq \cdots \geq \Delta_{d, 1}$. Note that the cyclic factorization $\mathbf{p}$ defined above is uniquely determined except for the special role of the first coordinate. We could conceivably arrive at a different cyclic factorization by working with the set of generators $\left\{\Delta_{i, \ell}: i \neq \ell\right\}$ for any $\ell \in\{2, \ldots, d\}$. In the calculations below we adopt the convention $\Delta_{i, i}=-1$. Note that we have

$$
\Delta_{i, \ell}=-\Delta_{i, 1} \Delta_{\ell, 1}^{-1}=\Delta_{i, 1} \frac{k 2^{d}}{\Delta_{\ell, 1}} .
$$

It follows that we have

$$
\ell<i \leq d \Rightarrow \Delta_{i, \ell}=-\frac{\Delta_{i, 1}}{\Delta_{\ell, 1}} \quad \text { and } \quad 1 \leq i<\ell \Rightarrow \Delta_{i, \ell}=\Delta_{i, 1} \cdot \frac{k 2^{d}}{\Delta_{\ell, 1}},
$$


and

$$
\left|\Delta_{\ell+1, \ell}\right| \leq \cdots \leq\left|\Delta_{d, \ell}\right| \leq\left|\Delta_{1, \ell}\right| \leq \cdots \leq\left|\Delta_{\ell-1, \ell}\right| .
$$

From this ordered collection we get the following sequence of factors (in the case $\ell=d$ the first two rows should be ignored):

$$
\begin{gathered}
\frac{-\Delta_{\ell+1, \ell}}{2}=\frac{\Delta_{\ell+1,1}}{2 \Delta_{\ell, 1}} \\
\ell+1 \leq j<d \Rightarrow \frac{\Delta_{j+1, \ell}}{2 \Delta_{j, \ell}}=\frac{\Delta_{j+1,1}}{2 \Delta_{j, 1}} \\
\frac{-\Delta_{1, \ell}}{2 \Delta_{d, \ell}}=\frac{\frac{k 2^{d}}{\Delta_{\ell, 1}}}{2 \frac{-\Delta_{d, 1}}{\Delta_{\ell, 1}}}=\frac{-k 2^{d}}{2 \Delta_{d, 1}} \\
1 \leq j<\ell-1 \Rightarrow \frac{\Delta_{j+1, \ell}}{2 \Delta_{j, \ell}}=\frac{\Delta_{j+1,1}}{2 \Delta_{j, 1}} \\
\frac{k 2^{d}}{2 \Delta_{\ell-1, \ell}}=\frac{\Delta_{\ell, 1}}{2 \Delta_{\ell-1,1}} .
\end{gathered}
$$

Thus, working with the generators $\left\{\Delta_{i, \ell}: i \neq \ell\right\}$ we arrive at the same cyclic factorization.

\section{References}

[1] L. Baumert, R. McEliece, E. Rodemich, H. Rumsey, R. Stanley, H. Taylor, A combinatorial packing problem, Computers in Algebra and Number Theory, Providence, American Mathematical Society, 1971, pp. 97-108.

[2] T. Bohman, R. Holzman, V. Natarajan, On the independence numbers of the cubes of odd cycles, manuscript.

[3] R. S. Hales, Numerical invariants and the strong product of graphs, Journal of Combinatorial Theory - B 15 (1973), 146-155.

[4] J. Körner and A. Orlitsky, Zero-error information theory, IEEE Transactions on Information Theory 44 (1998), 2207-2229.

[5] L. Lovász, On the Shannon capacity of a graph, IEEE Transactions on Information Theory 25 (1979), 1-7.

[6] C. E. Shannon, The zero-error capacity of a noisy channel, IRE Transactions on Information Theory 2 (1956), 8-19. 